\title{
The Phosphatase SHP-2 Activates HIF-1 $\alpha$ in Wounds In Vivo by Inhibition of 26S Proteasome Activity
}

\author{
Yvonn Heun 1,2, Katharina Grundler Groterhorst 1,3, Kristin Pogoda 1,2, Bjoern F Kraemer ${ }^{3}$, \\ Alexander Pfeifer ${ }^{4}$, Ulrich Pohl 1,2 and Hanna Mannell 1,2,5,* \\ 1 Walter Brendel Centre of Experimental Medicine, University Hospital, Ludwig-Maximilians-University, \\ Marchioninistr. 27, 81377 Munich, Germany \\ 2 Biomedical Center, Ludwig-Maximilians-University, Großhaderner Str. 9, 82152 Planegg, Germany \\ 3 Medizinische Klinik und Poliklinik I, Klinikum der Universität München, LMU, Marchioninistrasse 15, \\ 81377 Munich, Germany \\ 4 Institute of Pharmacology and Toxicology, Biomedical Center University of Bonn, Sigmund-Freud-Straße 25, \\ 53105 Bonn, Germany \\ 5 Hospital Pharmacy, Klinikum der Universität München, LMU, Marchioninistrasse 15, \\ 81377 Munich, Germany \\ * Correspondence: hanna.mannell@med.uni-muenchen.de; Tel.: +49-89-4400-44560
}

Received: 27 July 2019; Accepted: 5 September 2019; Published: 7 September 2019

check for updates

\begin{abstract}
Vascular remodeling and angiogenesis are required to improve the perfusion of ischemic tissues. The hypoxic environment, induced by ischemia, is a potent stimulus for hypoxia inducible factor $1 \alpha(\mathrm{HIF}-1 \alpha)$ upregulation and activation, which induce pro-angiogenic gene expression. We previously showed that the tyrosine phosphatase SHP-2 drives hypoxia mediated HIF- $1 \alpha$ upregulation via inhibition of the proteasomal pathway, resulting in revascularization of wounds in vivo. However, it is still unknown if SHP-2 mediates HIF-1 $\alpha$ upregulation by affecting $26 \mathrm{~S}$ proteasome activity and how the proteasome is regulated upon hypoxia. Using a reporter construct containing the oxygen-dependent degradation (ODD) domain of HIF- $1 \alpha$ and a fluorogenic proteasome substrate in combination with SHP-2 mutant constructs, we show that SHP-2 inhibits the 26S proteasome activity in endothelial cells under hypoxic conditions in vitro via Src kinase/p38 mitogen-activated protein kinase (MAPK) signalling. Moreover, the simultaneous expression of constitutively active SHP-2 (E76A) and inactive SHP-2 (CS) in separate hypoxic wounds in the mice dorsal skin fold chamber by localized magnetic nanoparticle-assisted lentiviral transduction showed specific regulation of proteasome activity in vivo. Thus, we identified a new additional mechanism of SHP-2 mediated HIF-1 $\alpha$ upregulation and proteasome activity, being functionally important for revascularization of wounds in vivo. SHP-2 may therefore constitute a potential novel therapeutic target for the induction of angiogenesis in ischemic vascular disease.
\end{abstract}

Keywords: SHP-2; tyrosine phosphatase; HIF-1; 26S proteasome; hypoxia; vascular remodeling; angiogenesis

\section{Introduction}

The transcription factor hypoxia inducible factor $1 \alpha(\mathrm{HIF}-1 \alpha)$ is involved in vascular remodeling and angiogenesis [1]. Ischemic cardiovascular disease is characterized by reduced tissue perfusion and reduced tissue oxygen partial pressure (hypoxia), which represent a strong stimulus for HIF- $1 \alpha$ activation [2]. HIF-1 $\alpha$ induces the expression of several angiogenic genes, such as vascular endothelial growth factor (VEGF), platelet derived growth factor (PDGF) or matrix metalloprotease 2 (MMP-2), which are potent inducers of angiogenesis and arteriogenesis [1,3]. It has therefore been the target of therapeutic strategies to increase tissue perfusion in ischemic limbs [4,5] and to improve wound 
healing [6,7]. During normoxic conditions, HIF- $1 \alpha$ is hydroxylated on prolyl residues within its oxygen-dependent degradation (ODD) domain, leading to von-Hippel-Lindau protein (pVHL) dependent ubiquitinylation and subsequent degradation by the proteasome [8,9]. Hypoxia inhibits the proteasomal degradation of HIF- $1 \alpha$ by inhibition of the prolyl hydroxylation domain containing enzymes (PHD), resulting in stabilization of HIF- $1 \alpha$ and subsequent accumulation within the cell $[8,9]$. Additionally, it has been shown that HIF-1 $\alpha$ may be degraded by the protease calpain [10].

The tyrosine phosphatase SHP-2 has been demonstrated by us to positively influence angiogenesis in vitro and in vivo [11-13] and may constitute an interesting future therapeutic target within this context. In an earlier study we demonstrated that SHP-2 is important for HIF- $1 \alpha$ stabilisation and activity during hypoxia, resulting in enhanced hypoxia induced HIF-1 $\alpha$ dependent revascularisation of wounds in vivo [12]. Further, we showed that SHP-2 activates the Src kinase upon hypoxia, which in turn influenced HIF- $1 \alpha$ prolyl hydroxylation [12]. Finally, we found that the impaired HIF- $1 \alpha$ accumulation observed upon SHP-2 inactivation could be rescued by treatment with a PHD inhibitor as well as the proteasome inhibitors MG132 and Epoxomicin [12]. However, while our data indicated that SHP-2 influences HIF- $1 \alpha$ accumulation during hypoxia by affecting the activity of the PHD, thus determining the proteasomal degradation of HIF- $1 \alpha$, we did not investigate whether the effect may additionally be caused by regulation of proteasome activity.

The $26 \mathrm{~S}$ proteasome is responsible for the degradation of ubiquitinylated proteins and consists of a $20 \mathrm{~S}$ core particle and two $19 \mathrm{~S}$ regulatory particles [14]. The 20S core particle exhibits three peptidase activities (caspase-like; C-L, trypsin-like; T-L and chymotrypsin-like; CT-L), which are responsible for the cleavage of protein substrates. Ubiquitinylated proteins are recognized and bind to the $19 \mathrm{~S}$ regulatory particle, which is in addition responsible for the ATP-dependent unfolding of the substrate protein and the opening of the $20 \mathrm{~S}$ core particle, where degradation occurs [14]. The activity of the $26 \mathrm{~S}$ proteasome has been shown to be regulated by phosphorylations via threonine/serine kinases as well as tyrosine kinases on several subunits of the $19 S$ and $20 S$ particles [15]. However, not very much is known regarding the role of phosphatases in regulating $26 \mathrm{~S}$ proteasome activity. Moreover, its regulation during hypoxia still needs to be investigated.

In this study, we investigated the activity of the $26 \mathrm{~S}$ proteasome during hypoxia and the connection to SHP-2 in endothelial cells in vitro and in hypoxic wounds in vivo. We found SHP-2 to inhibit the $26 \mathrm{~S}$ proteasome activity in hypoxic cells, as assessed by measuring $26 \mathrm{~S}$ peptidase activity as well as the accumulation of a HIF-1-ODD-Luc reporter construct. Importantly, we demonstrated that SHP-2 regulates $26 \mathrm{~S}$ proteasome activity in hypoxic wounds in vivo.

\section{Results}

\subsection{SHP-2 Inactivation Leads to Increased Proteasome Dependent HIF-1 $\alpha$ Degradation during Hypoxia}

As HIF-1 $\alpha$ has been shown to be degraded by the proteasome as well as calpain [10], we first investigated whether this is also true in endothelial cells upon hypoxia. Treatment with the specific proteasome inhibitor epoxomicin or the calpain inhibitor MG101, respectively, resulted in an increase in HIF-1 $\alpha$ protein accumulation (Figure 1A). We previously observed that SHP-2 inactivation impaired HIF-1 $\alpha$ accumulation [12], which was rescued by treatment with proteasome inhibitors. We now additionally investigated the involvement of calpain. As seen in Figure 1B, overexpression of a dominant negative SHP-2 (SHP-2 CS) impaired HIF-1 $\alpha$ accumulation under hypoxic conditions compared to cells overexpressing SHP-2 wildtype (WT). Treatment with the calpain inhibitor MG101 could not rescue this effect. Having observed a proteasome dependent [12] but calpain independent degradation of HIF-1 $\alpha$ upon SHP-2 inactivation, we next investigated $26 \mathrm{~S}$ proteasome activity in endothelial cells under hypoxia. For this, we induced the lentiviral expression of a construct containing the oxygen-dependent degradation (ODD) domain of HIF-1 $\alpha$ [16], which guides its proteasomal degradation upon ubiquitinylation [3], fused to a luciferase gene (HIF1-ODD-Luc) with simultaneous expression of mCherry, in endothelial cells. The expression of HIF1-ODD-Luc thus 
inversely correlates with $26 \mathrm{~S}$ proteasome activity and has been used as a measure of proteasome activity before [16]. First, to test its function in endothelial cells, luciferase activity upon treatment with proteasome inhibitors or hypoxia was measured $72 \mathrm{~h}$ after transduction with HIF1-ODD-Luc. As seen in Figure 1C, treatment with the proteasome inhibitors Bortezomib and MG132 as well as hypoxic exposure $(4 \mathrm{~h})$ significantly increased the accumulation of HIF1-ODD-Luc, reflecting the inhibition of the $26 \mathrm{~S}$ proteasome. The transduction of endothelial cells with a control reporter construct lacking the HIF-1 $\alpha$ ODD (Ctrl-Luc) showed a strong constitutive expression of luciferase, as expected, which did not differ between normoxic and hypoxic conditions (Figure S1). Expression of dominant negative SHP-2 (CS) impaired HIF1-ODD-Luc accumulation compared to SHP-2 WT expressing cells upon hypoxia, thus demonstrating an increase in $26 \mathrm{~S}$ proteasome activity (Figure 1D).

A
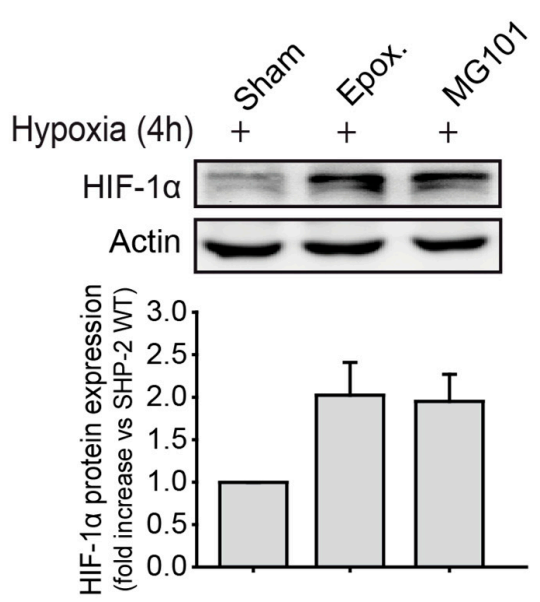

C

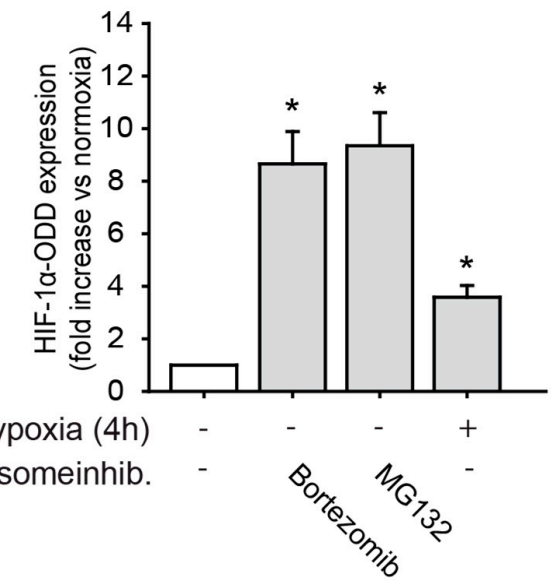

B
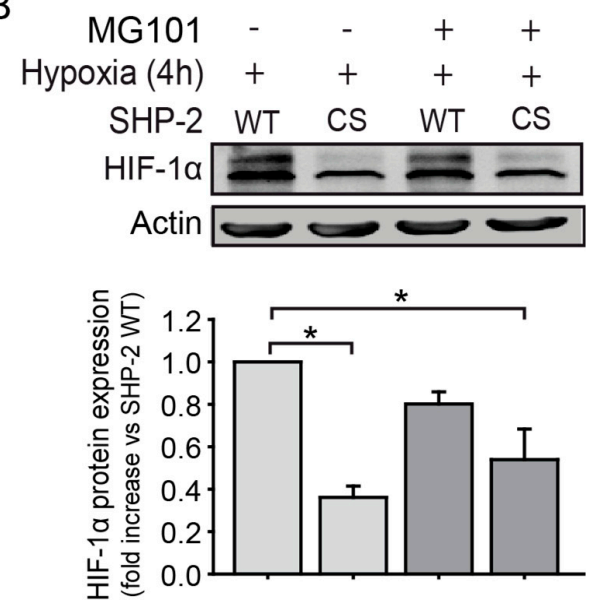

$\mathrm{D}$

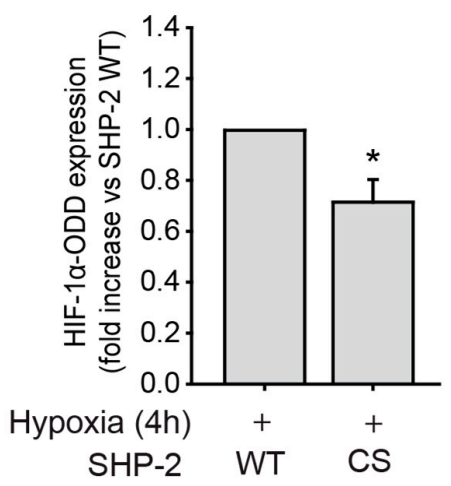

Figure 1. SHP-2 inactivation enhances proteasome dependent hypoxia inducible factor $1 \alpha$ (HIF-1 $\alpha$ ) degradation in endothelial cells during hypoxia. (A) HIF-1 $\alpha$ protein levels were increased during hypoxia upon inhibition of the 26S proteasome (Epoxomicin, $10 \mu \mathrm{M}$ ) as well as calpain (MG101, $5 \mu \mathrm{M}$ ) $(n=3)$. Graph underneath blot shows the protein band densities normalized to $\beta$-actin. (B) Expression of dominant negative SHP-2 (CS) prevents hypoxic HIF-1 $\alpha$ protein upregulation, which could not be rescued by calpain inhibition (MG101, $5 \mu \mathrm{M} ;{ }^{*} p<0.05 ; n=3$ ). Graph underneath blot shows the protein band densities normalized to $\beta$-actin. (C) The reporter construct HIF1-ODD-Luc accumulated upon inhibition of the proteasome (Bortezomib $64 \mathrm{nM}$; MG132 $10 \mu \mathrm{M}$ ) during normoxia as well as under only hypoxia ( $\left.{ }^{*}<<0.05 ; n=16-25\right)$, confirming the specificity of the reporter constructs. (D) Expression of dominant negative SHP-2 (CS) increased HIF-1 $\alpha$ degradation by the proteasomal pathway, as detected by lower expression of HIF1-ODD-Luc $\left({ }^{*} p<0.05, n=17\right)$. 


\subsection{SHP-2 Regulates Proteasomal Degradation of HIF-1 $\alpha$ in Hypoxic Wounds In Vivo}

As we previously found SHP-2 inactivation to prevent HIF- $1 \alpha$ accumulation and activity in endothelial cells upon hypoxia, resulting in impaired wound healing angiogenesis in vivo [12] and, as we now observed that SHP-2 inactivation increases $26 \mathrm{~S}$ proteasomal activity under hypoxia in endothelial cells in vitro, we investigated the proteasomal activity in vivo. For this, HIF1-ODD-Luc or Ctrl-Luc were expressed in wounds of the dorsal skin of mice by localized magnetic nanoparticles-assisted lentiviral transduction (Figure S3). By using lentiviruses (LV) coupled to magnetic nanoparticles (MNP) and the application of an external magnetic field, the simultaneous transduction of three individual wounds in the same animal can be achieved [12]. As seen in Figure 2A and Figure S2B, HIF1-ODD-Luc only accumulated in the malperfused wound and not after transduction of healthy tissue, confirming that the wound is hypoxic and that proteasome activity is higher in normoxic tissues. As a positive control, wounds were transduced with Ctrl-Luc, which causes a continuous strong expression of luciferase, as this construct does not contain the HIF- $1 \alpha$ ODD domain. Next, we performed co-transductions of individual wounds in the same animal with HIF1-ODD-Luc and the different SHP-2 constructs, to investigate the influence of SHP-2 on proteasome activity in vivo. Whereas the expression of inactive SHP-2 CS in hypoxic wounds significantly inhibited HIF1-ODD-Luc accumulation via increased proteasome activity, introduction of the constitutively active SHP-2 E76A (Glu76 to Ala76) enhanced the HIF1-ODD-Luc protein accumulation compared to SHP-2 WT expressing wounds (Figure 2B and Figure S2C). This indicates that SHP-2 regulates HIF- $1 \alpha$ stabilization and accumulation in hypoxic wounds by decreasing $26 \mathrm{~S}$ proteasome activity.

A
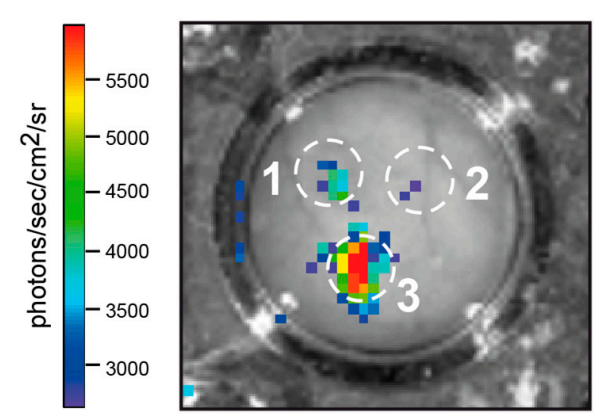

1. HIF1-ODD-Luc w. wound

2. HIF1-ODD-Luc wo. wound

3. ctrl.-Luc w. wound

B

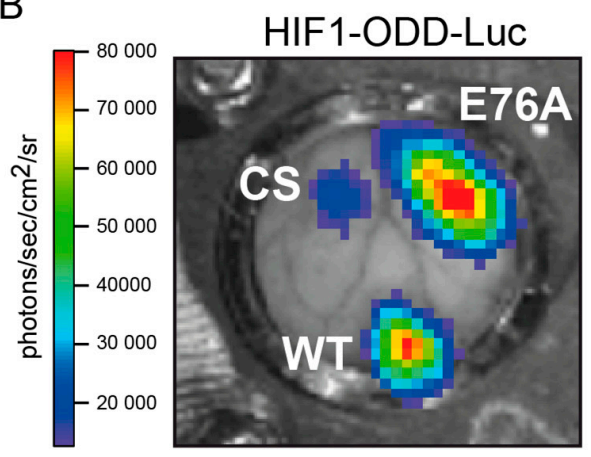

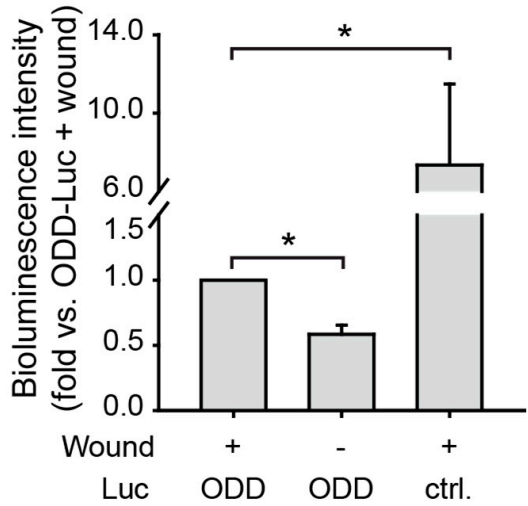

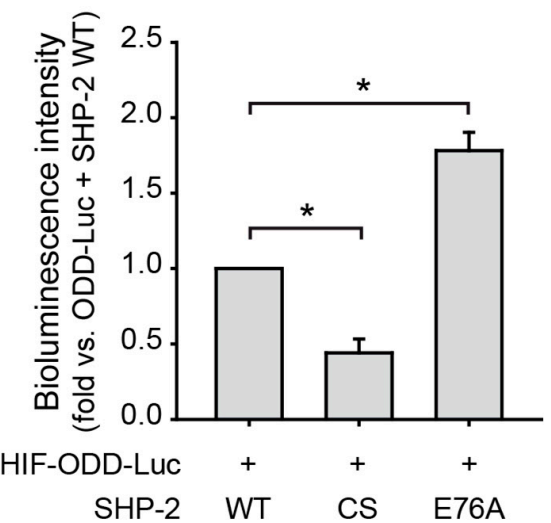

Figure 2. SHP-2 inactivation induces HIF-1 $\alpha$ degradation via the proteasome pathway in hypoxic wounds in vivo. (A) Wounds in the same dorsal skin fold chamber in mice were simultaneously transduced with HIF1-ODD-Luc or Ctrl-Luc lacking HIF1-ODD using site directed lentiviral magnetic 
targeting [12]. HIF1-ODD-Luc was expressed in wounds (1) but was degraded by the proteasome in healthy tissue (2), demonstrating the specificity of the lentiviral constructs and that the wounds are hypoxic $\left({ }^{*} p<0.05 ; n=\right.$ three animals). (3) Ctrl-Luc lacking the HIF-ODD domain was therefore constitutively expressed in the wound $\left({ }^{*} p<0.05 ; n=\right.$ three animals). (B) Wounds in the same dorsal skin fold chamber in mice were simultaneously co-transduced with HIF1-ODD-Luc and different SHP-2 constructs. While HIF1-ODD-Luc accumulated in hypoxic wounds upon transduction with SHP-2 WT, expression of dominant negative SHP-2 (CS) impaired this, demonstrating an increased 26S proteasomal activity ( ${ }^{*} p<0.05 ; n=3-4$ animals). Expression of constitutively active SHP-2 (E76A) further enhanced HIF1-ODD-Luc accumulation, demonstrating enhanced inhibition of 26S proteasome activity ( ${ }^{*} p<0.05 ; n=3-4$ animals). Wounding was performed the day after implantation of the dorsal skin fold chamber. Transduction of wounds was performed $24 \mathrm{~h}$ after wounding and measurements of luciferase activity were performed eight days after transduction (see also Figure S2A).

\subsection{The Proteasomal Degradation of HIF-1 $\alpha$ is Dependent on Src Kinase and p38 MAPK Activation}

In a former study, we could show that SHP-2 induces HIF-1 $\alpha$ expression via a Src kinase dependent mechanism in endothelial cells upon hypoxia [12]. We thus hypothesized that the observed effect of SHP-2 on 26 S proteasome activity and HIF-1 $\alpha$ stabilization in this study may be mediated by Src as well. To test this, endothelial cells were transduced with the HIF1-ODD-Luc reporter construct and treated with the pharmacological Src inhibitor PP2 upon hypoxia. Whereas hypoxia induced the stabilization, and thus accumulation, of HIF1-ODD-Luc, representing a decrease in proteasome activity, Src inhibition significantly impaired this response (Figure 3A). Src kinases have been demonstrated to induce the activation of p38 MAPK during hypoxia [17]. Thus, we next explored whether this was the case in endothelial cells. The hypoxia induced HIF-1 $\alpha$ accumulation was prevented upon inhibition of p38 MAPK (Figure 3B). Moreover, hypoxia induced the phosphorylation and thus activation of p38 MAPK and this was abrogated when treating cells with the Src kinase inhibitor PP2 (Figure 3C). Moreover, treatment with the p38 MAPK inhibitor SB203580 increased proteasome activity, reflected by reduced HIF1-ODD-Luc accumulation (Figure 3D). Finally, the phosphorylation of p38 MAPK was impaired in endothelial cells expressing SHP-2 CS and enhanced in cells expressing the constitutively active SHP-2 E76A compared to the expression of SHP-2 WT upon hypoxia (Figure 3E).

\subsection{SHP-2 Activity Inhibits the Chymotrypsin-Like Activity of the 26 S Proteasome upon Hypoxia}

As previously published data from our group showed that treatment with epoxomicin and MG132, which are both inhibitors of the CT-L activity of the $26 \mathrm{~S}$ proteasome [18], rescued the low HIF- $1 \alpha$ protein level caused by SHP-2 inactivation [12], we next detected the 26S CT-L proteolytic activity in endothelial cells under hypoxia. We used experimental conditions optimized to investigate proteolytic protease activity of the $26 \mathrm{~S}$ proteasome as previously described [19]. For this, cells expressing SHP-2 WT were exposed to hypoxia and the chymotrypsin-like (CT-L) activity of the 26S proteasome was assessed by a specific fluorogenic proteasome substrate (Suc-LLVY-AMC). Hypoxia significantly reduced CT-L activity (Figure 4A and Figure S4), and endothelial cells expressing constitutively active SHP-2 E76A exhibited an even lower 26S CT-L activity upon hypoxia compared to SHP-2 WT expressing cells (Figure 4B), indicating a downregulation of the CT-L proteolytic activity of the $26 \mathrm{~S}$ proteasome. 
A

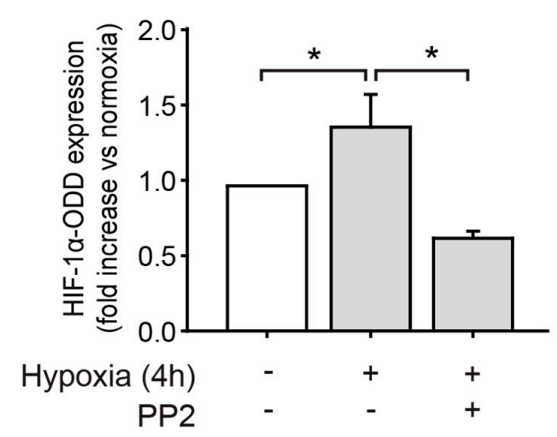

C
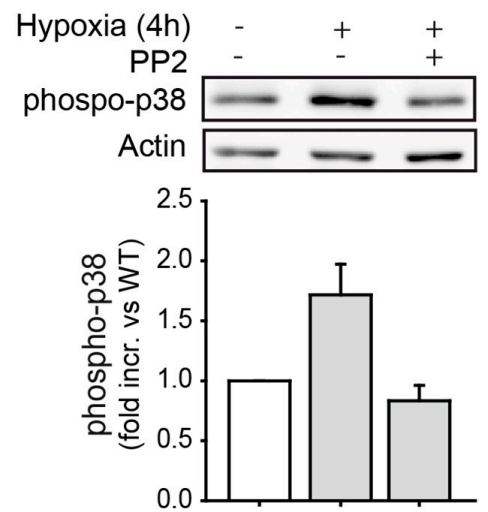

$E$
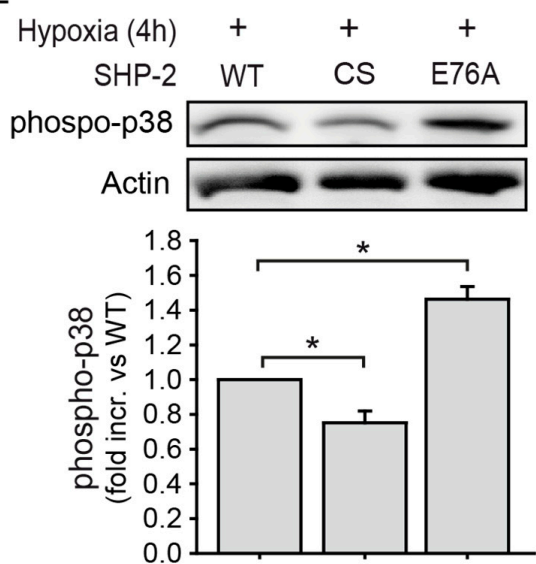

B

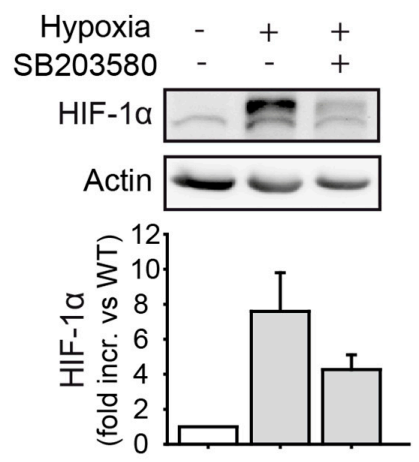

D

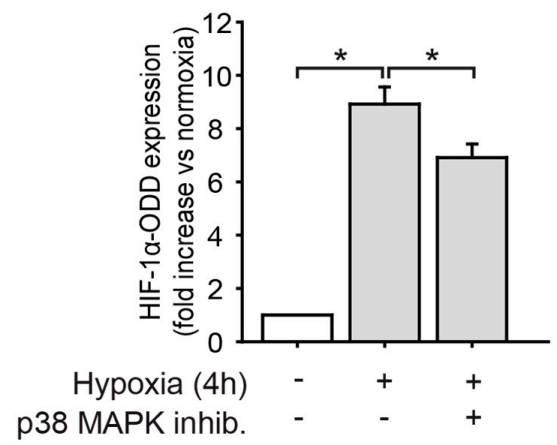

Figure 3. The proteasomal HIF- $1 \alpha$ degradation is dependent on Src kinase and p38 mitogen-activated protein kinase (MAPK) signaling. (A) Whereas hypoxia inhibited $26 \mathrm{~S}$ proteasome activity in endothelial cells, as seen by increased expression of HIF1-ODD-Luc, inhibition of Src kinase (PP2, $100 \mathrm{nM})$ reversed this $\left({ }^{*} p<0.05 ; n=9\right)$. (B) Inhibition of p38 MAPK (SB203580, $\left.10 \mu \mathrm{M}\right)$ in endothelial cells impaired hypoxia induced HIF-1 $\alpha$ expression $(n=6)$. (C) Src kinase inhibition $(\mathrm{PP} 2,100 \mathrm{nM})$ reduced hypoxia induced p38 MAPK activation $(n=2)$. (D) p38 MAPK inhibition (SB203580, $10 \mu \mathrm{M})$ increased 26S proteasome activity, as measured by a lower level of HIF1-ODD-Luc reporter expression $\left({ }^{*} p<0.05 ; n=4\right)$. (E) Expression of dominant negative SHP-2 (CS) impaired hypoxia induced p38 MAPK phosphorylation, whereas expression of constitutively active SHP-2 (E76A) enhanced this compared to SHP-2 WT ${ }^{*} p<0.05$; $n=4)$. Graphs underneath blots show the protein band densities normalized to $\beta$-actin. 
A

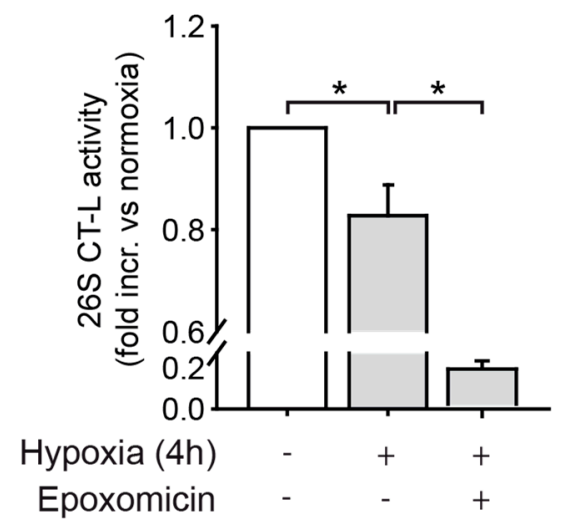

B

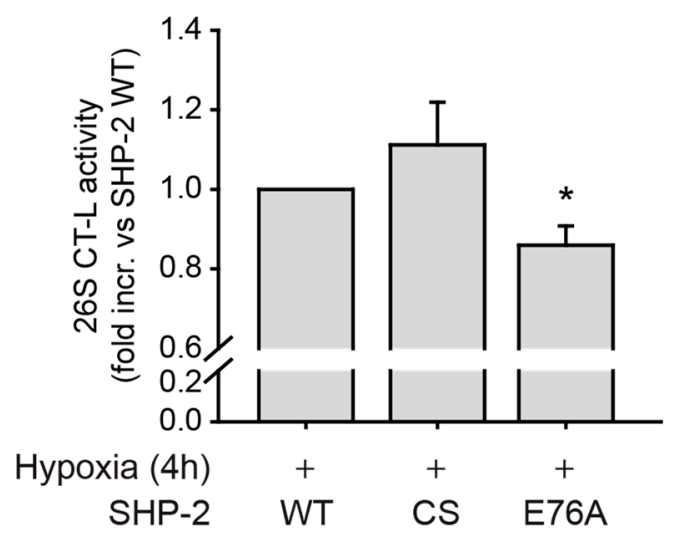

Figure 4. SHP-2 inhibits $26 \mathrm{~S}$ chymotrypsin-like (CT-L) proteasomal activity during hypoxia. (A) The CT-L activity of the $26 \mathrm{~S}$ proteasome was decreased upon hypoxia $\left({ }^{*} p<0.05, n=11\right)$, as measured by the fluorogenic substrate Suc-LLVY-AMC. Treatment with epoxomicin $(10 \mu \mathrm{M})$ was used as a positive control and effectively inhibited the CT-L proteolytic activity of the 26S proteasome $\left({ }^{*} p<0.05 ; n=3\right)$. (B) Expression of constitutively active SHP-2 (E76A) also significantly impaired the CT-L activity of the $26 \mathrm{~S}$ proteasome compared to SHP-2 WT during hypoxia $\left({ }^{*} p<0.05, n=9\right.$ ), whereas the expression of dominant negative SHP-2 (CS) showed a tendency towards increased CT-L activity $(n=9)$.

\section{Discussion}

Vascular remodeling and angiogenesis are important for maintaining tissue perfusion upon ischemia [2]. We previously demonstrated that the tyrosine phosphatase SHP-2 drives hypoxia mediated HIF-1 $\alpha$ upregulation, resulting in revascularization of wounds in vivo [12]. Here, we show that this is achieved by SHP-2 dependent inhibition of $26 \mathrm{~S}$ proteolytic activity via Src kinase/p38 MAPK signalling.

In a former study, we demonstrated that SHP-2 promotes HIF- $1 \alpha$ stabilization and activity in endothelial cells during hypoxia as well as revascularization of hypoxic wounds in vivo by increasing HIF- $1 \alpha$ activity, resulting in higher expression of VEGF, MMP-2 and PDGF [12]. Moreover, we showed SHP-2 to negatively influence the proteasomal degradational pathway, as the impaired HIF- $1 \alpha$ expression seen upon SHP-2 inactivation was rescued by inactivation of the proteasome as well as the PHD [12]. While these results indicate that SHP-2 influences the prolylhydroxylation of HIF-1 $\alpha$ and in this way its proteasomal degradation, we further investigated here the mechanisms of SHP-2 and its regulation of proteasomal activity. The data obtained in this study confirm our previous findings and additionally reveal a second mechanism of promoting HIF- $1 \alpha$ upregulation by directly affecting $26 \mathrm{~S}$ proteasomal activity. Inactivation of SHP-2 prevented the cellular accumulation of HIF1-ODD-Luc, which inversely correlated with $26 \mathrm{~S}$ proteasomal activity, in endothelial cells in vitro. The in vivo relevance of SHP-2 mediated proteasome regulation was confirmed in hypoxic wounds of intact animals using co-transduction of HIF1-ODD-Luc and SHP-2 constructs. Of note, expression of constitutively active SHP-2 further enhanced HIF1-ODD-Luc levels, demonstrating an increased inhibition of the 26S proteasome. This correlates well with the impaired 26S CT-L proteolytic activity measured in cells with constitutive SHP-2 activation. Thus, we conclude that SHP-2 activity negatively affects proteasomal activity during wound healing under hypoxic conditions in vivo.

As we previously found SHP-2 dependent Src kinase activation to be involved in HIF-1 $\alpha$ upregulation during hypoxia [12], we now investigated if it is also important for proteasomal activity. Indeed, Src integrity was crucial for $26 \mathrm{~S}$ proteasomal activity and in addition, was important for activation of the 338 MAPK upon hypoxia. p38 MAPK, in turn, positively affected HIF- $1 \alpha$ upregulation while negatively influencing $26 \mathrm{~S}$ proteasomal activity, a mechanism which was promoted by SHP-2. These results are not only in accordance with but additionally extend the study from Lee et al. 
performed in HeLa cells, demonstrating that p38 MAPK negatively affects $26 \mathrm{~S}$ proteolytic activity also in endothelial cells [20]. This inhibition was further shown to be due to the phosphorylation of Thr-273 on the subunit Rpn2 in the $19 S$ regulatory particle [20]. Whether this is the case during hypoxia, remains to be investigated. Posttranslational phosphorylation of the $26 \mathrm{~S}$ proteasome has repeatedly been shown to regulate its enzymatic activities [15]. Several stimuli, such as stress, metabolic changes, and growth factor signalling have been demonstrated to induce its phosphorylation [15]. Here, we identified hypoxia as a novel stimulus of $26 \mathrm{~S}$ proteasomal inactivation, particularly the CT-L activity. In addition, we found the caspase-like (C-L) activity to be deprived upon hypoxia (Figure S4). Intriguingly, we measured an increased trypsin-like (T-L) activity during hypoxia, suggesting that the different proteolytic activities of the $26 \mathrm{~S}$ proteasome may be differentially regulated. One may hypothesize that the differential regulation of activities is involved in substrate specificity of proteasomal degradation. However, more investigations are needed to elucidate the mechanisms behind this. The observed reduction in CT-L and C-L activity, however, is supported by the fact that the $26 \mathrm{~S}$ proteasome relies on the activity of ATPases in the 195 regulatory particle for substrate de-ubiquitinylation, unfolding and 20S gate opening and activation [15], which do not function upon ATP deprivation, as is the case during hypoxia. Moreover, we observed that inhibition of Src kinase/p38 MAPK signalling rescued proteasomal activation. Additionally, an inhibition of $26 \mathrm{~S}$ proteasomal activity via direct dephosphorylation of subunits by SHP-2 was not investigated here and has to be the focus of further studies. However, this is per se possible, as Zong et al. demonstrated the phosphatase PP2A to negatively affect $20 \mathrm{~S}$ proteolytic activity via dephosphorylation [21]. Nevertheless, this is to our knowledge the first study to report a regulation of $26 \mathrm{~S}$ proteolytic activity by SHP- 2 .

In summary, we were able to further characterize the mechanism behind the regulation of hypoxia mediated HIF-1 $\alpha$ upregulation by SHP-2, which is essential for revascularisation of malperfused wounds. Moreover, we demonstrate for the first time that the $26 \mathrm{~S}$ proteasomal activity is regulated by SHP-2 during hypoxia in vitro and is functionally relevant in vivo. We show that SHP-2 not only inhibits the proteasomal degradation pathway by influencing HIF- $1 \alpha$ prolylhydroxylation [12] but in addition by directly inhibiting $26 \mathrm{~S}$ proteolytic activity via p38 MAPK (for a summary of our findings, see Figure 5). We thus believe that SHP-2 is important for HIF- $1 \alpha$ upregulation in vitro and in wounds in vivo by inhibition of the proteasomal pathway via activation of Src kinase/p38 MAPK signalling. Together with our previous results, this regulation may be achieved by redundant and or additive pathways, involving external regulators of the proteasome (PHD and pVHL activation), and directly via the CT-L activity of the $26 \mathrm{~S}$ proteasome. Finally, our findings confirm SHP-2 to be essential for hypoxic HIF-1 $\alpha$ upregulation in vivo. SHP-2 may therefore constitute a novel therapeutic target in ischemic vascular disease, aiming for the revascularization of ischemic tissues. 


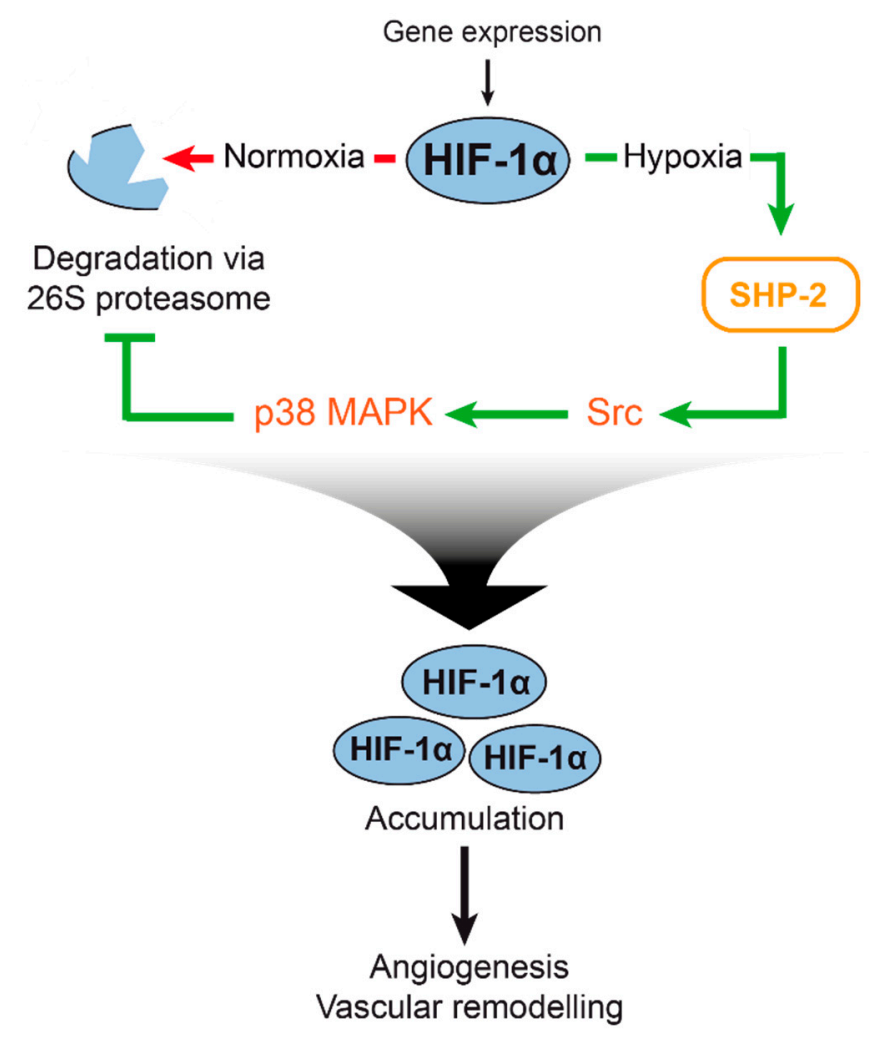

Figure 5. Illustration of $26 \mathrm{~S}$ proteasome regulation and HIF-1 $\alpha$ accumulation by SHP-2 in hypoxia. HIF- $1 \alpha$ is constitutively expressed but degraded by the proteasomal pathway during hypoxia (left). Upon hypoxia (right), the degradation of HIF- $1 \alpha$ is inhibited. In our previous study, we found SHP-2 to influence the prolyl hydroxylation of HIF-1 $\alpha$, which targets it for degradation [12]. In this study, we found a second mechanism of SHP-2 mediated HIF- $1 \alpha$ upregulation: SHP-2 positively affects Src kinase and p38 MAPK activity during hypoxia, which in turn negatively influences the activity of the $26 \mathrm{~S}$ proteasome. As a consequence, HIF- $1 \alpha$ is stabilized and accumulates in the cell, promoting hypoxic angiogenesis and vascular remodeling.

\section{Materials and Methods}

\subsection{Antibodies and Chemicals}

Rabbit phospho-p38 MAPK (Thr180/Tyr182) (D3F9) XPTM (\#4511) and $\beta$-Actin (13E5) antibodies were from Cell Signaling Technology, Frankfurt am Main, Germany. HIF- $1 \alpha$ clone H1 $\alpha 67$, HIF-1 $\alpha$ clone EP1215Y, anti-mouse and -rabbit horseradish peroxidase-conjugated secondary antibodies were from Merck Millipore, Darmstadt, Germany. Src-Inhibitor PP2 was purchased from Sigma-Aldrich (\#P0042), Darmstadt, Germany. p38 MAPK inhibitor (SB203580), MG132 and MG101 were from Tocris, Wiesbaden-Nordenstadt, Germany. Bortezomib and Epoxomicin were from Calbiochem. All other chemicals were from Sigma-Aldrich, Darmstadt, Germany.

\subsection{Human Microvascular Endothelial Cell (HMEC) Culture}

Human dermal microvascular endothelial cells (HMEC) [22] were cultivated as described earlier [23]. In detail, HMEC were cultivated in DMEM (Sigma-Aldrich, Darmstadt, Germany) containing 10\% fetal calf serum (Biochrom, Berlin, Germany) and 1\% Penicillin-Streptomycin (Sigma-Aldrich, Darmstadt, Germany) and kept in an incubator with $5 \% \mathrm{CO}_{2}$ at $37^{\circ} \mathrm{C}$. 


\subsection{Lentiviral Constructs and Transductions}

Wild type (WT) SHP-2 and catalytically inactive mutant SHP-2 CS (Cys459 to Ser459) plasmid vectors were kind gifts from the Bennett laboratory [24]. The constitutively active SHP-2 E76A (Glu76 to Ala) was generated as previously described [12]. The lentiviral constructs containing the above mentioned cDNAs were generated as described earlier [12] and lentiviral particles were produced as described elsewhere [25]. Flow cytometry of transduced HEK293T cells was used to determine the biological titer as previously described [25]. The FUW-ODD-Luc-mCherry lentiviral plasmid (referred to as HIF1-ODD-Luc in this manuscript) and the control vector FUW-Luc-mCherry (referred to as Ctrl-Luc in this manuscript) were kindly provided by Kimbrel et al. [16] and packaged into lentiviral particles as previously described [25]. The ODD-Luc insert encodes a reporter fusion protein consisting of the oxygen-dependent domain (ODD) from hypoxia-inducible factor 1a (HIF-1a) and firefly luciferase, inversely reflecting proteasomal activity, with simultaneous expression of mCherry after a self-cleavage 2A site. Lentiviral transduction of HMECs was carried out using a multiplicity of infection (MOI) of five. Lentiviral particles were diluted in Hank's solution and applied onto subconfluent HMEC. After incubation for $4-6 \mathrm{~h}$ at $37^{\circ} \mathrm{C}$, culture medium was added. The next day the medium was changed, and cells were left $72 \mathrm{~h}$ before assaying.

\subsection{Hypoxia Treatment}

For hypoxia treatment, HMECs were incubated in cultivation media in a hypoxia chamber (Cell Systems, Troisdorf, Germany) at $\mathrm{pO}_{2} 8 \pm 2 \mathrm{mmHg}$ equivalent to a $\mathrm{O}_{2}$ concentration of $1 \pm 0.2 \%$ for $4 \mathrm{~h}$ as previously described [26]. To reach these experimental hypoxic conditions, the chamber was flooded for four minutes with 15-20 L/min with an anoxic gas mixture $\left(5 \% \mathrm{CO}_{2}, 95 \% \mathrm{~N}_{2}\right)$. After hypoxia, the media was discarded, and cells quickly washed once with phosphate buffered saline and lysed for further processing.

\subsection{In Vitro 26S Proteasome Activity}

The 26S CT-L proteasome activity was measured as previously described [19]. In detail, HMEC were subjected to hypoxia $(4 \mathrm{~h})$, rinsed with cold phosphate buffered saline supplemented with calcium (PBS+) and lysed at $4{ }^{\circ} \mathrm{C}$ with lysis buffer ( $1 \mathrm{mM}$ DTT, $1 \times$ Roche PhosphoStop Tablet in homogenizing buffer containing $20 \mathrm{mM}$ HEPES, $1 \mathrm{mM} \mathrm{MgCl} 2,150 \mathrm{mM} \mathrm{NaCl}$, and $0.5 \mathrm{mM}$ EDTA). $10 \mu \mathrm{g}$ protein in assay buffer containing 1mM DTT, $50 \mu \mathrm{M}$ ATP and $100 \mu \mathrm{M}$ Suc-LLVY-AMC (R\&D systems, Wiesbaden, Germany) in homogenizing buffer was measured at $37^{\circ} \mathrm{C}$ with excitation wavelength $339 \mathrm{~nm}$ and emission wavelength $439 \mathrm{~nm}$ for $2 \mathrm{~h}$.

\subsection{In Vitro Luciferase Assay}

HMEC were seeded in 24-well plates and transduced the following day. Indicated inhibitor treatments and hypoxia was performed $72 \mathrm{~h}$ post transduction and cells were lysed on ice for $30 \mathrm{~min}$ ( $250 \mathrm{mM}$ Tris base $\mathrm{pH7} .8,0.1 \%$ Triton-X). Then, $10 \mu \mathrm{L}$ of cell lysate were transferred to a black 96-well plate in triplicates and $100 \mu \mathrm{L}$ luciferin assay buffer $\left(60 \mathrm{mM}\right.$ DTT, $10 \mathrm{mM} \mathrm{MgSO}_{4}, 1 \mathrm{mM}$ ATP, $25 \mathrm{mM}$ Glycil-Glycin, $0.3 \mathrm{mM}$ D-Luciferin) were added to each well. Bioluminescence was detected using the Spectrafluor (Tecan, Männedorf, Switzerland) with a one-second integration time and normalized to protein concentrations.

\subsection{In Vivo Transduction and Luciferase Imaging}

Animal studies were conducted in accordance with the German animal protection law and approved by the district government of upper Bavaria (Regierung von Oberbayern, approval reference number AZ55.2-1-54-2532-172-13). The investigation conforms to the Guide for the Care and Use of Laboratory Animals published by the US National Institutes of Health (NIH Publication No. 85-23, revised 1996). 16-20-week old male and female C57 BL6/J mice (Charles River) were anesthetized 
( $5 \mathrm{mg} / \mathrm{kg}$ midazolam, $0.5 \mathrm{mg} / \mathrm{kg}$ medetomidin, and $0.05 \mathrm{mg} / \mathrm{kg}$ fentanyl) and the dorsal skinfold chamber was implanted as described before [27]. Wounds were introduced using a hot probe as previously described $24 \mathrm{~h}$ after dorsal skinfold chamber implantation [12]. Proteasomal activity in avascular wounds in the dorsal skin of mice was detected by localized magnetic nanoparticles-assisted transduction of individual wounds in the same animal with HIF1-ODD-Luc (FUW-ODD-Luc-mCherry) and Ctrl-Luc (FUW-Luc-mCherry) lentiviral vectors as well as co-transductions of with the ODD-Luc vector and SHP-2 WT, CS and E76A lentiviral vectors, respectively, as previously described [12]. Luciferase activity was detected eight days after transduction by application of $3 \mathrm{mg} / \mathrm{mL}$ D-Luciferin directly to the imaging window of the dorsal skinfold chamber. Bioluminescence was imaged using the IVIS ${ }^{\circledR}$ spectrum in vivo imaging system from PerkinElmer at medium binning and exposure times between five and 10 minutes. Signal intensities were quantified using the Fiji software.

\subsection{Immunoblotting}

Cell lysates were prepared and subjected to SDS-PAGE followed by western blotting as previously described [28]. Protein band intensities were measured using the Hokawo software (Hamamatsu, Herrsching, Germany) and normalized to the respective $\beta$-Actin protein bands, which was used as equal loading control.

\subsection{Statistical Analysis}

Data are presented as means \pm SEM. Statistical analyses were performed with Sigma Plot 10.0. The Student's t-test was used for comparisons between two groups of normal distributed data, rank-sum test was performed for comparisons of two groups of not normally distributed data. The one-way analysis of variance (one-way ANOVA) was performed for multiple comparisons. Differences were considered significant at an error probability level of $p<0.05$.

Supplementary Materials: Supplementary materials can be found at http://www.mdpi.com/1422-0067/20/18/4404/s1.

Author Contributions: Conceptualization, supervision and project administration: H.M.; Investigation: Y.H., K.G.G., K.P., H.M.; Methodology: A.P., Y.H., H.M.; Visualization: H.M., Y.H. Writing-original draft preparation: H.M., B.F.K., U.P., A.P.; Funding Acquisition: H.M., Y.H.

Funding: This research was funded by Dr. Kleist-Stiftung and Friedrich Baur Stiftung.

Acknowledgments: The authors would like to thank Andrew Kung at Harvard Medical School, Boston, USA for kindly providing us with the lentiviral constructs HIF1-ODD-Luc (FUW-ODDLuc-mCherry) and Ctrl-luc (FUW-wtLuc-mCherry) [16].

Conflicts of Interest: The authors declare no conflict of interest and the funders had no role in the design of the study; in the collection, analyses, or interpretation of data; in the writing of the manuscript, or in the decision to publish the results.

\section{Abbreviations}

Ctrl-Luc Lentiviral control luciferase reporter construct lacking the HIF-1 $\alpha$ ODD

HIF1-ODD-Luc Lentiviral construct containing the oxygen-dependent degradation (ODD) domain of

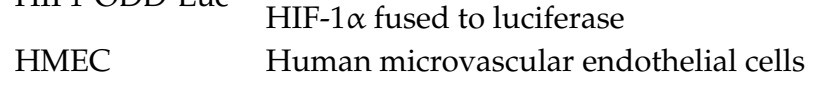

SHP-2 Src homology domain containing tyrosine phosphatase 2

SHP-2 WT SHP-2 wildtype construct

SHP-2 CS Dominant negative SHP-2 construct where Cys459 was exchanged to Ser459

SHP-2 E76A Constitutively active SHP-2 construct where Glu76 was exchanged to Ala76 


\section{References}

1. Semenza, G.L. Targeting hypoxia-inducible factor 1 to stimulate tissue vascularization. J. Investig. Med. Off. Publ. Am. Fed. Clin. Res. 2016, 64, 361-363. [CrossRef] [PubMed]

2. Rey, S.; Semenza, G.L. Hypoxia-inducible factor-1-dependent mechanisms of vascularization and vascular remodelling. Cardiovasc. Res. 2010, 86, 236-242. [CrossRef] [PubMed]

3. Krock, B.L.; Skuli, N.; Simon, M.C. Hypoxia-induced angiogenesis: Good and evil. Genes Cancer 2011, 2, 1117-1133. [CrossRef] [PubMed]

4. Sarkar, K.; Fox-Talbot, K.; Steenbergen, C.; Bosch-Marce, M.; Semenza, G.L. Adenoviral transfer of HIF-1alpha enhances vascular responses to critical limb ischemia in diabetic mice. Proc. Natl. Acad. Sci. USA 2009, 106, 18769-18774. [CrossRef] [PubMed]

5. Patel, T.H.; Kimura, H.; Weiss, C.R.; Semenza, G.L.; Hofmann, L.V. Constitutively active HIF-1alpha improves perfusion and arterial remodeling in an endovascular model of limb ischemia. Cardiovasc Res. 2005, 68, 144-154. [CrossRef] [PubMed]

6. Botusan, I.R.; Sunkari, V.G.; Savu, O.; Catrina, A.I.; Grunler, J.; Lindberg, S.; Pereira, T.; Yla-Herttuala, S.; Poellinger, L.; Brismar, K.; et al. Stabilization of HIF-1alpha is critical to improve wound healing in diabetic mice. Proc. Natl. Acad. Sci. USA 2008, 105, 19426-19431. [CrossRef] [PubMed]

7. Mace, K.A.; Yu, D.H.; Paydar, K.Z.; Boudreau, N.; Young, D.M. Sustained expression of Hif- $1 \alpha$ in the diabetic environment promotes angiogenesis and cutaneous wound repair. Wound Repair Regen. 2007, 15, 636-645. [CrossRef]

8. Walshe, T.E.; D'Amore, P.A. The role of hypoxia in vascular injury and repair. Annu Rev. Pathol. 2008, 3, 615-643. [CrossRef]

9. Bilton, R.L.; Booker, G.W. The subtle side to hypoxia inducible factor (HIFalpha) regulation. Eur. J. Biochem. 2003, 270, 791-798. [CrossRef]

10. Zhou, J.; Kohl, R.; Herr, B.; Frank, R.; Brune, B. Calpain mediates a von Hippel-Lindau protein-independent destruction of hypoxia-inducible factor-1alpha. Mol. Biol. Cell 2006, 17, 1549-1558. [CrossRef]

11. Mannell, H.; Hellwig, N.; Gloe, T.; Plank, C.; Sohn, H.Y.; Groesser, L.; Walzog, B.; Pohl, U.; Krötz, F. Inhibition of the tyrosine phosphatase SHP-2 suppresses angiogenesis in vitro and in vivo. J. Vasc. Res. 2008, 45, 153-163. [CrossRef] [PubMed]

12. Heun, Y.; Pogoda, K.; Anton, M.; Pircher, J.; Pfeifer, A.; Woernle, M.; Ribeiro, A.; Kameritsch, P.; Mykhaylyk, O.; Plank, C.; et al. HIF-1alpha Dependent Wound Healing Angiogenesis In Vivo Can Be Controlled by Site-Specific Lentiviral Magnetic Targeting of SHP-2. Mol. Ther. 2017, 25, 1616-1627. [CrossRef] [PubMed]

13. Rieck, S.; Heun, Y.; Heidsieck, A.; Mykhaylyk, O.; Pfeifer, A.; Gleich, B.; Mannell, H.; Wenzel, D. Local anti-angiogenic therapy by magnet-assisted downregulation of SHP2 phosphatase. J. Control. Release 2019, 305, 155-164. [CrossRef]

14. Jang, H.H. Regulation of Protein Degradation by Proteasomes in Cancer. J. Cancer Prev. 2018, 23, $153-161$. [CrossRef] [PubMed]

15. Guo, X.; Huang, X.; Chen, M.J. Reversible phosphorylation of the 26S proteasome. Protein Cell 2017, 8, $255-272$. [CrossRef] [PubMed]

16. Kimbrel, E.A.; Davis, T.N.; Bradner, J.E.; Kung, A.L. In vivo pharmacodynamic imaging of proteasome inhibition. Mol. Imaging 2009, 8, 140-147. [CrossRef]

17. Thobe, B.M.; Frink, M.; Choudhry, M.A.; Schwacha, M.G.; Bland, K.I.; Chaudry, I.H. Src family kinases regulate p38 MAPK-mediated IL-6 production in Kupffer cells following hypoxia. Am. J. Physiol. 2006, 291, C476-C482. [CrossRef]

18. Wojcik, C.; Di Napoli, M. Ubiquitin-proteasome system and proteasome inhibition: New strategies in stroke therapy. Stroke 2004, 35, 1506-1518. [CrossRef]

19. Grundler, K.; Rotter, R.; Tilley, S.; Pircher, J.; Czermak, T.; Yakac, M.; Gaitzsch, E.; Massberg, S.; Krotz, F.; Sohn, H.Y.; et al. The proteasome regulates collagen-induced platelet aggregation via nuclear-factor-kappa-B (NFkB) activation. Thromb. Res. 2016, 148, 15-22. [CrossRef]

20. Lee, S.H.; Park, Y.; Yoon, S.K.; Yoon, J.B. Osmotic stress inhibits proteasome by p38 MAPK-dependent phosphorylation. J. Biol. Chem. 2010, 285, 41280-41289. [CrossRef] 
21. Zong, C.; Gomes, A.V.; Drews, O.; Li, X.; Young, G.W.; Berhane, B.; Qiao, X.; French, S.W.; Bardag-Gorce, F.; Ping, P. Regulation of murine cardiac 20S proteasomes: Role of associating partners. Circ. Res. 2006, 99, 372-380. [CrossRef] [PubMed]

22. Ades, E.W.; Candal, F.J.; Swerlick, R.A.; George, V.G.; Summers, S.; Bosse, D.C.; Lawley, T.J. HMEC-1: Establishment of an immortalized human microvascular endothelial cell line. J. Investig. Derm. 1992, 99, 683-690. [CrossRef] [PubMed]

23. Mannell, H.K.; Pircher, J.; Chaudhry, D.I.; Alig, S.K.; Koch, E.G.; Mettler, R.; Pohl, U.; Krötz, F. ARNO regulates VEGF-dependent tissue responses by stabilizing endothelial VEGFR-2 surface expression. Cardiovasc. Res. 2012, 93, 111-119. [CrossRef] [PubMed]

24. Kontaridis, M.I.; Liu, X.; Zhang, L.; Bennett, A.M. Role of SHP-2 in fibroblast growth factor receptor-mediated suppression of myogenesis in C2C12 myoblasts. Mol. Cell. Biol. 2002, 22, 3875-3891. [CrossRef] [PubMed]

25. Hofmann, A.; Wenzel, D.; Becher, U.M.; Freitag, D.F.; Klein, A.M.; Eberbeck, D.; Schulte, M.; Zimmermann, K.; Bergemann, C.; Gleich, B.; et al. Combined targeting of lentiviral vectors and positioning of transduced cells by magnetic nanoparticles. Proc. Natl. Acad. Sci. USA 2009, 106, 44-49. [CrossRef] [PubMed]

26. Alig, S.K.; Stampnik, Y.; Pircher, J.; Rotter, R.; Gaitzsch, E.; Ribeiro, A.; Wornle, M.; Krötz, F.; Mannell, H. The Tyrosine Phosphatase SHP-1 Regulates Hypoxia Inducible Factor-1alpha (HIF-1alpha) Protein Levels in Endothelial Cells under Hypoxia. PLoS ONE 2015, 10, e0121113. [CrossRef] [PubMed]

27. Mannell, H.; Pircher, J.; Fochler, F.; Stampnik, Y.; Räthel, T.; Gleich, B.; Plank, C.; Mykhaylyk, O.; Dahmani, C.; Wornle, M.; et al. Site directed vascular gene delivery in vivo by ultrasonic destruction of magnetic nanoparticle coated microbubbles. Nanomedicine 2012, 8, 1309-1318. [CrossRef]

28. Krötz, F.; Engelbrecht, B.; Buerkle, M.A.; Bassermann, F.; Bridell, H.; Gloe, T.; Duyster, J.; Pohl, U.; Sohn, H.Y. The tyrosine phosphatase, SHP-1, is a negative regulator of endothelial superoxide formation. J. Am. Coll. Cardiol. 2005, 45, 1700-1706.

(C) 2019 by the authors. Licensee MDPI, Basel, Switzerland. This article is an open access article distributed under the terms and conditions of the Creative Commons Attribution (CC BY) license (http://creativecommons.org/licenses/by/4.0/). 